\title{
Reply to the Comments of Dikpati et al.
}

\author{
A. R. Choudhuri ${ }^{1}$, D. Nandy ${ }^{2}$, and P. Chatterjee ${ }^{1}$ \\ 1 Department of Physics, Indian Institute of Science, Bangalore-560012, India \\ e-mail: arnab@physics.iisc.ernet.in \\ 2 Department of Physics, Montana State University, Bozeman, MT 59717, USA
}

Received 25 February 2005 / Accepted 21 March 2005

\begin{abstract}
We respond to Dikpati et al.'s criticism of our recent solar dynamo model. A different treatment of the magnetic buoyancy is the most probable reason for their different results.
\end{abstract}

Key words. Sun: activity - Sun: magnetic fields

\section{Introduction}

Dikpati et al. (2005, hereafter DRGM) have commented on a recent paper by us (Chatterjee et al. 2004, hereafter CNC) presenting a solar dynamo model. Their criticisms broadly fall under two categories. Firstly, they point out that they are unable to reproduce our results (see Sects. 3.2, 3.3 and 3.5 of their paper). Secondly, they have raised some concerns about the basics of our model, including the name "circulation-dominated dynamo" (see Sects. 3.1, 3.4, 4.1 and 4.2 of DRGM).

\section{Possible reason for divergent results}

Unfortunately there is an error in Eq. (11) of CNC, which gives the stream function of the velocity field. The correct stream function used in our code is

$$
\begin{aligned}
& \psi r \sin \theta=\psi_{0}\left(r-R_{p}\right) \sin {\left[\frac{\pi\left(r-R_{p}\right)}{\left(R_{\odot}-R_{p}\right)}\right]\left\{1-\mathrm{e}^{-\beta_{1} \theta^{\epsilon}}\right\} } \\
&\left\{1-\mathrm{e}^{\beta_{2}(\theta-\pi / 2)}\right\} \mathrm{e}^{-\left(\left(r-r_{0}\right) / \Gamma\right)^{2}}
\end{aligned}
$$

with the parameters $\beta_{1}=1.5, \beta_{2}=1.8, \epsilon=2.0000001$, $r_{0}=\left(R_{\odot}-R_{b}\right) / 4.0, \Gamma=3.47 \times 10^{8} \mathrm{~m}, \gamma=0.95$, and $m=3 / 2$. It was mistakenly printed as

$$
\begin{array}{r}
\psi r \sin \theta=\psi_{0}\left(r-R_{p}\right) \sin \left[\frac{\pi\left(r-R_{p}\right)}{\left(R_{\odot}-R_{p}\right)}\right]\left\{1-\mathrm{e}^{-\beta_{1} r \theta^{\epsilon}}\right\} \\
\left\{1-\mathrm{e}^{\beta_{2} r(\theta-\pi / 2)}\right\} \mathrm{e}^{-\left(\left(r-r_{0}\right) / \Gamma\right)^{2}}
\end{array}
$$

with $\beta_{1}=1.36 \times 10^{-8} \mathrm{~m}^{-1}$ and $\beta_{2}=1.63 \times 10^{-8} \mathrm{~m}^{-1}$, while the other parameters were given as quoted above. We had used the form (2) earlier (e.g., Choudhuri \& Dikpati 1999). Subsequently we found that (1) gives more satisfactory results; this form has therefore been used by Nandy \& Choudhuri (2002) and by CNC. We regret the mistake and are grateful to DRGM for their efforts in reproducing our results, which made us aware of this. However, we do not think that the slightly different meridional circulation caused the difference between our results. Our code gives qualitatively similar results with both (1) and (2). Another mistake in CNC is that the value of $r_{\mathrm{TCZ}}$ appearing in (13) is given as $0.95 R_{\odot}$ rather than $0.975 R_{\odot}$ which is the value used.

We believe that DRGM's result differs from ours because of their different handling of magnetic buoyancy. We have made some runs by switching off our buoyancy algorithm and using the non-local buoyancy of Dikpati \& Charbonneau (1999), i.e. multiplying $\alpha$ not by the local $B$ but by $B\left(r=0.7 R_{\odot}, \theta\right)$. In contrast to the results presented in CNC, we get multi-lobed patterns, as shown in Fig. 1. This figure is very similar to Fig. 3 of DRGM. The period for this solution is $6.1 \mathrm{yr}$. In the buoyancy algorithm of CNC, only when $B$ is larger than a critical value $B_{c}$ above $r=0.71 R_{\odot}$, the toroidal field erupts to the surface and contributes to the generation of the poloidal field. This happens only at sufficiently low latitudes. In contrast, the non-local buoyancy algorithm makes even a weak toroidal field $B\left(r=0.7 R_{\odot}, \theta\right)$ at high latitudes contribute to the generation of poloidal field at the surface - a clearly unphysical mechanism which upsets the dynamo solution completely. The period of the dynamo also becomes shorter because the toroidal field starts generating poloidal field while still at mid-latitudes, instead of having to be advected all the way to low latitudes.

We plan to make our code available to the public within a few months, with the parameters used to generate the solution presented in Sects. 3.1 and 4 of CNC.

\section{Response to other criticisms}

\subsection{Is our dynamo model circulation-dominated?}

In Sect. 3.1 of DRGM question the name "circulationdominated" we had given to our dynamo. Let us explain our point of view. We have $\mathrm{d} \Omega / \mathrm{d} r$ positive at the lower latitudes, 

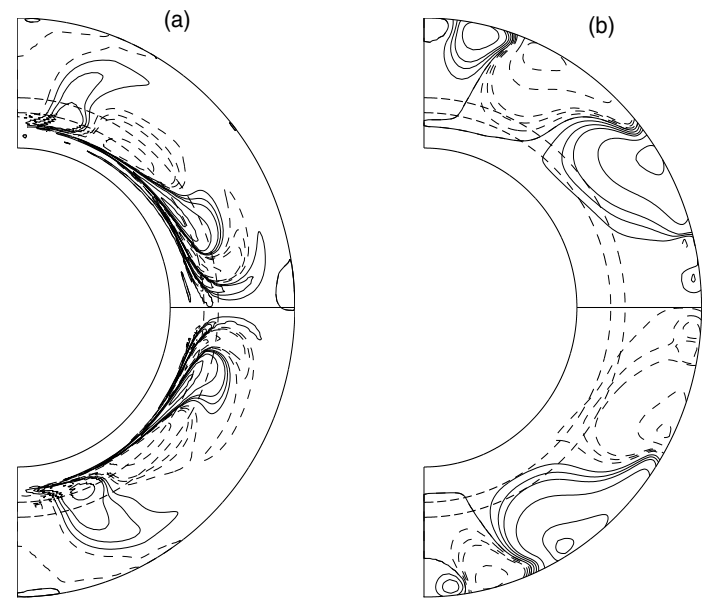

Fig. 1. Toroidal (left) and poloidal (right) magnetic fields in the dynamo solution obtained with the non-local buoyancy formalism of Dikpati \& Charbonneau (1999).

and the $\alpha$ coefficient is also positive. It is well-known that the dynamo wave should then propagate poleward. Still the toroidal field below the solar convection zone (SCZ) moves equatorward, because it is advected through a region where the diffusivity has a low value $\eta_{\mathrm{RZ}}=2.2 \times 10^{8} \mathrm{~cm}^{2} \mathrm{~s}^{-1}$ and it essentially remains frozen in the fluid. Even if we take a rather low value of $d=10^{4} \mathrm{~km}$ for the thickness of the layer through which the toroidal field is advected, the diffusion time $d^{2} / \eta_{\mathrm{RZ}}$ is about $144 \mathrm{yr}$ - much larger than the time scale of advection by the meridional circulation. That is why we felt that "circulation-dominated" is an appropriate name.

\section{2. $T F / P F$ ratios}

In Sect. 3.4 of DRGM, our model is criticized in that polar fields of order $2 \mathrm{kG}$ are needed to generate $100 \mathrm{kG}$ toroidal fields. The answer to this can be found in Sects. 2-3 of Choudhuri (2003). The dynamo equation deals with the mean magnetic field. On the other hand, flux tube rise simulations suggested a magnetic field of $100 \mathrm{kG}$ only inside the flux tubes - the mean field being much less. Choudhuri (2003, Sect. 2) estimated that a circulation-dominated dynamo can stretch a polar field of $10 \mathrm{G}$ to a maximum toroidal field of only about $10 \mathrm{kG}$. So the mean toroidal field can be at most of this value. The toroidal field has to be highly intermittent, with the value of $100 \mathrm{kG}$ occuring only in isolated regions. This is an entirely consistent scenario.

\subsection{Different diffusivities for PF and TF}

Since there are not separate conducting fluids for toroidal and poloidal fields, DRGM argue in Sect. 4.2 that assuming different diffusivities at the same point is implausible. However, the diffusivities entering the dynamo equation are not "physical" diffusivities, but effective diffusivities which arise from an averaging procedure and describe how the mean fields evolve. We believe that the magnetic field at the base of SCZ looks as sketched in Fig. 3 of Choudhuri (2003), with

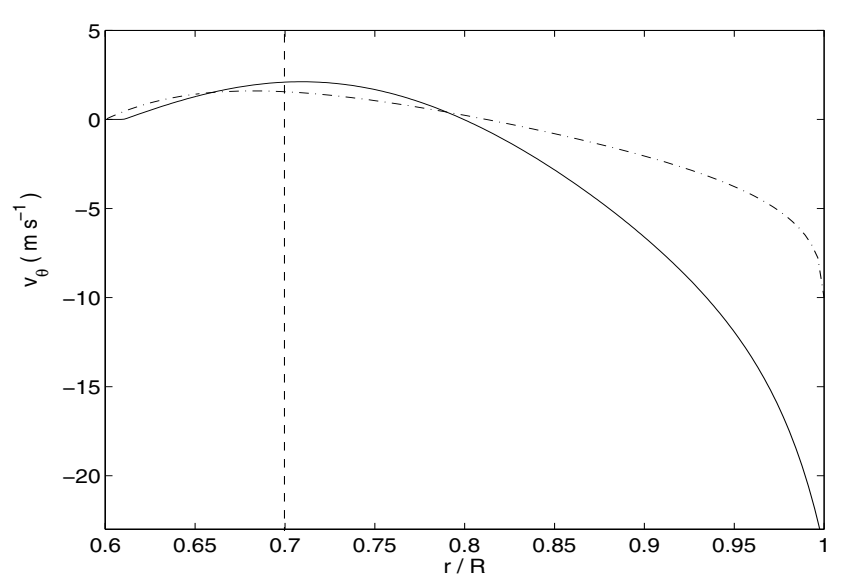

Fig. 2. Plot of $v_{\theta}(r)$ at $\theta=45^{\circ}$, as used by CNC (solid) and by Dikpati $\&$ Charbonneau (1999) (dashed-dotted; see their Fig. 1c).

the flux concentrations stretching along the $\phi$ direction. Turbulent diffusivity is obviously suppressed within these flux concentrations, making the magnetic field $B_{\phi}$ in these regions rather immune to turbulent diffusivity. How do we capture the information in the dynamo equation that diffusivity is suppressed within $B_{\phi}$ concentrations having sizes smaller than the scale of our mean field? We felt that taking a smaller diffusivity for $B_{\phi}$ is one way of handling this issue.

\subsection{Penetration depth of meridional circulation}

DRGM argue in Sect 4.1 that the meridional circulation could not penetrate below the SCZ which we require. It may be pointed out that Dikpati \& Charbonneau (1999) produced their best dynamos with a deeply penetrating circulation, without clearly mentioning that this was essential to obtain good models. Nandy \& Choudhuri (2002) were the first who asserted clearly that a penetrating flow is required for a satisfactory circulation-dominated solar dynamo. Figure 2 shows the profiles $v_{\theta}(r)$ at $\theta=45^{\circ}$ used by us and by Dikpati \& Charbonneau (1999). DRGM seem to conclude that our model has a velocity of $10 \mathrm{~ms}^{-1}$ at $r=0.6 R_{\odot}$; but this is not the case, see Fig. 3 of CNC.

We agree that the meridional circulation could not penetrate much into a stable region where there is no overshooting or turbulence. However, in our current ignorance, we cannot rule out the possibility of enough overshooting and turbulence throughout the tachocline below the SCZ and a circulation penetrating through this region.

\section{References}

Chatterjee, P., Nandy, D., \& Choudhuri, A. R. 2004, A\&A, 427, 1019 Choudhuri, A. R. 2003 Sol. Phys., 215, 31

Choudhuri, A. R., \& Dikpati, M. 1999, Sol. Phys., 184, 61

Dikpati, M., \& Charbonneau, P. 1999, ApJ, 518, 508

Dikpati, M., Rempel, M., Gilman, P. A., \& MacGregor, K. B. 2005, A\&A, 437, 699

Nandy, D., \& Choudhuri, A. R. 2002, Science, 296, 1671 